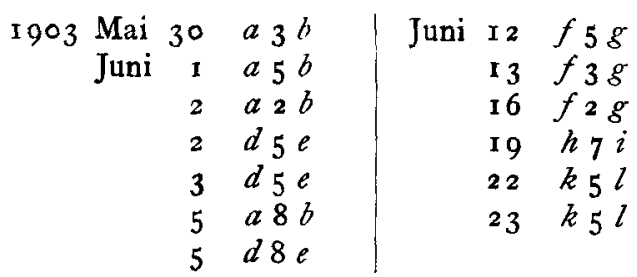

(324) Bamberga.

\begin{tabular}{rl|ll} 
r903 Juli 28 & $m 5 n$ \\
$3 \mathrm{I}$ & $=0$ \\
Aug. 4 & $p 5 q$ & $\begin{array}{l}\text { Aug. 2 I } \\
\text { Sept. 2 }\end{array}$ & $t 5 u$
\end{tabular}

(270) Anahita.

I 903 Juli $31 \quad v 5 w$

3. Vom 1 3. April bis zu Ende des Jahres r 903 sind alle Beobachtungen (mit Ausnahme des 20. April und 27. Mai) mit Hilfe des Registrierapparates von Fueß gemacht worden.

4. Die Sternpositionen aus den Katalogen Par. und $\operatorname{Rad}_{3}$ sind auf das System der AG. nach Auwers reduziert.

(24) Themis. März 16, I8, I9. Bilder schlecht. Beobachtete den Planeten mit Schwierigkeit. - März 2 r. Bilder schön.

(29) Amphitrite. März r9, 20, 31. Bilder sehr schlecht. - März 25. Bei einer Position des Mikrometers. - April 3, 9, 2o. Wind, Fernrohr zittert. - April 20. Bilder sebr schlecht.

(16) Psyche. Durch Wolken, kaum sichtbar.

(39) Laetitia. Mai r 9. Wind, Fernrohr zittert.

(17) Thetis. Mai I 9. Bilder schlecht. Zweite Hälfte der Beobachtung durch Wolken. -- Mai 22, 27. Bilder schlecht. - Juni r bis 23. Himmel hell (Dämmerung während der ganzen Nacht). - Juni I 2. Helligkeitsunterschied der Vergleichsterne in $\mathrm{BD} .=0^{\mathrm{m}} \cdot 3$, ist aber ungefähr $\mathrm{I}^{\mathbf{m}} \circ$. Juni 22 und 23. Sehe den Planeten mit Schwierigkeit.

(324) Bamberga. Juli 28 . Vergleichstern ist doppelt, beobachtete den Schwerpunkt. - Aug. I3. Beobachtung durch Wolken unterbrochen, bei einer Position des Mikrometers. - Aug. 24. Bilder schlecht. - Aug. 30. Wind, Fernrohr zittert. - Sept. 2. Bilder sehr schlecht.

(37) Fides. Jan. 29. Planet kaum sichtbar. - Febr. 6. Himmel neblig. Bilder sehr schlecht. schein.

(3 13) Chaldaea. Durch Strati, kaum sichtbar. Mond-

Komet I903 IV. Juni 25. Komet kaum sichtbar (Dämmerung). - Juni 30 . Fernrohr zittert, Vorbeifahren von Wagen. - Juli r. Ende bei Tagesanbruch. - Juli 2. Ende durch Wolken. - Juli 4. Ende bei Tagesanbruch. - Juli 7. Durch Wolken; Kern verwaschen. - Juli 8. Bilder sehr schlecht. - Juli 9. Ende bei Tagesanbruch. Vorbeifahren von Wagen. - Juli Io. Himmel neblig. - Juli I I erste Beobachtung, Juli 12 zweite Beobachtung, Juli I 4, erste Beobachtung, mikrometrische Messung der beiden Koordinaten. - Juli I I. Koma dichter nach SSW. Stellung des Beobachters unbequem (Komet nahe dem Zenit). - Juli I 2. Wind, Fernrohr zittert. Cirri. - Juli I4. Wind. Stellung des Beobachters sehr unbequem. Schweif kaum sichtbar. PW. I $77^{\circ}$. - Juli 16 . Stellung des Beobachters sehr unbequem. Durch Wolken. - Juli 18 . Schweif PW. $163^{\circ}$. Wind. - Juli r 9. Schweif PW. r68. Wind. - Juli 21. Schweif PW. I $28^{\circ}$. Durch Wolken. - Juli 22. Schweif PW. I1 $3^{\circ}$. Vorbeifahren von Wagen. Kern verwaschen. - Juli 24.

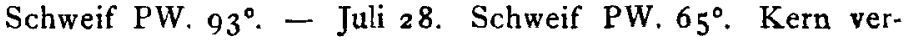
waschen. - Juli 30 . Schweif PW. $7^{\circ}$. - Aug. I. Schweif PW. $78^{\circ}$. Bilder schlecht. - Aug. 2. Kern schlecht sichtbar. - Aug. 4. Schweif PW. $89^{\circ}$. Kern sehr verwaschen. Himmel neblig. - Aug. 9. Ende durch Wolken. Himmel neblig. - Aug. ×3. Schweif (kaum sichtbar) PW. $52^{\circ}$. Wolken. - Aug. 17. Schweif PW. 47 . Beobachtung durch Wolken unterbrochen. - Aug. I8. Bilder schwach, Kern verwaschen. - Aug. 2 I. Wind. Fernrohr zittert. - Aug. 24. Bilder sehr schlecht, Komet erscheint wie eine Scheibe von 20" Durchmesser. Nahe dem Horizont.

Beobachter Assistent M. Iwanowski.

Kasan, 1904 Mai I 2.

\title{
Osservazioni della cometa 1904 I
}

fatte col micrometro anulare al refrattore equatoriale di 8 pollici del R. Osserv. di Brera in Milano.

\begin{tabular}{|c|c|c|c|c|c|c|c|c|c|c|}
\hline 1904 & T.m. Milano & $\Delta \alpha$ & $\Delta \delta$ & Cf. & $\alpha$ app. & $\log p \cdot \Delta$ & $\delta$ app. & $\log p \cdot \Delta$ & Red.ad l. app. & $*$ \\
\hline g. 15 & $10^{\mathrm{h}} 12^{\mathrm{m}} 3^{2^{\mathrm{s}}}$ & $+1^{m} 43^{s} \cdot 58$ & $\mathbf{2}^{\prime} 55^{\prime}$ & I 6 & I $5^{\mathrm{h}} 6^{\mathrm{m}} 55^{\mathrm{s}} \cdot 9 \mathrm{I}$ & & +5 & & $23+3$ & 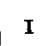 \\
\hline I 6 & 102951 & -049.42 & -1026.32 & IO & 210.53 & $9.264 n$ & +57 1838.7 & $0.225 \mathrm{n}$ & $+2.23+3.4$ & 2 \\
\hline I 7 & II 1726 & +311.3 & +259.40 & 9 & 145720.26 & 7.462 & +572851.5 & $0.269 n$ & $19+3.9$ & 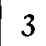 \\
\hline I 8 & IO $475^{\circ}$ & $+\mathrm{I} I 4.1$ & +47.50 & 9 & I 45245 & $8.874 \mathrm{n}$ & +573735 & $8 n$ & $18+4 \cdot 3$ & 4 \\
\hline iug. 4 & I0 3321 & -235.04 & +0 & I 4 & I3 4I 26.94 & $9 \cdot 528$ & +5744 I9.4 & $0.137 \mathrm{n}$ & $+1.54+9.3$ & 5 \\
\hline
\end{tabular}

Posizioni medie delle stelle di paragone.

\begin{tabular}{|c|c|c|c|}
\hline * & $\alpha 1904.0$ & $\delta \times 904.0$ & Autorità \\
\hline $\mathbf{I}$ & I $5^{\mathrm{h}} 5^{\mathrm{m}}$ I0.10 & $+57^{\circ} \quad 4^{\prime} 14^{\prime \prime}: 5$ & AG. Hels. 8240 \\
\hline 2 & 257.72 & $+5729 \quad 1.6$ & 8227 \\
\hline 3 & $\times 454$ & +572548.2 & 8 I 83 \\
\hline
\end{tabular}

Milano, 24 Giugno 1904.

\begin{tabular}{|c|c|c|c|}
\hline$*$ & $\alpha I 904.0$ & $d 1904.0$ & Autorità \\
\hline 4 & $\mathrm{I} 4^{\mathrm{h}} 5 \mathrm{I}^{\mathrm{m}} 29^{\mathrm{s}} \cdot 6 \mathrm{I}$ & $+57^{\circ} 33^{\prime} 23^{\prime \prime} 3$ & AG. Hels. $8 \mathrm{I}$ \\
\hline 5 & I $344 \quad 0.44$ & +57449.0 & $\gg 7709$ \\
\hline
\end{tabular}

Luigi Gabba. 